\title{
PUBLICACIONES FEMINISTAS EN EL ECUADOR: CARACOLA Y EL ÁGORA DE LAS MUJERES
}

\author{
MARIA CUVI-SÁNCHEZ \\ Corporación Promoción de la Mujer
}

\begin{abstract}
Resumen: Este ensayo se refiere a Caracola y El Ágora de las Mujeres, dos publicaciones feministas que circulan en El Ecuador. La primera es una revista impresa y trimestral que apareció en marzo de 2001; la segunda es una página que se distribuyó por correo eléctrico durante el 2002 y el 2003.En el ensayo se plantean los objetivos de estas publicaciones, se describe la acogida que han tenido entre la audiencia que han captado, y los desafios que ambos medios de comunicación enfrentan. Dos son las preocupaciones principales de la autora: Cómo elevar la audibilidad de los discursos feministas en el medio ecuatoriano y cómo erosionar el pensamiento androcéntrico que domina las ciencias, la literatura y el arte en ese país.
\end{abstract} Palabras Clave: publicaciones feministas, políticas feministas, movimiento de mujeres

Caracola' y El Ágora de las Mujeres son dos publicaciones feministas que circulan en el Ecuador. La primera, una revista impresa trimestral, apareció en marzo de 2001; la segunda, una página que se distribuye por correo electrónico, en agosto de 2002. En este ensayo relato la corta vida de estas dos experiencias a la vez que lanzo al aire algunos desafíos que ambos medios de comunicación enfrentarán en el futuro inmediato. Tuve la fortuna de participar en el Primer Encuentro Internacional de Publicaciones Feministas realizado en Florianópolis en noviembre de 2003, donde conocí experiencias editoriales que nos llevan años de ventaja, y reconocí en éstas los principales obstáculos que deben vencer Caracola y El Ágora. Me siento menos sola desde entonces.

Caracola nace para llenar un vacío: la ausencia de publicaciones feministas en el Ecuador. Raquel Rodas, su directora, la fundó a su vuelta de España con el propósito de compartir lo aprendido y motivar a las mujeres a que expresasen sus pensamientos, su sentir y sus experiencias: escritoras, artistas, teóricas feministas y todas aquéllas que a través de su pluma estén dispuestas a invertir el mandato de ver, oír y callar, apunta Raquel. Es una revista que intenta despertar la sensibilidad estética de las mujeres. En palabras de su directora, constituye una alternativa de formación feminista que rompe la hegemonía del enfoque de género introducido por las agencias de desarrollo y del feminismo de la igualdad que sustenta las débiles intervenciones del Estado ecuatoriano. La revista abre la posibilidad de conocer otras corrientes feministas, especialmente la de la diferencia. En sus páginas se difunde el pensamiento de teóricas como María Milagros Rivera, Adriana Rich, Luisa Muraro, Lucy Irigaray. Una sección de Caracola está dedicada 
a recuperar la genealogía de las ecuatorianas, valorarlas, y dar cabida a escritoras noveles que no tienen posibilidades de escribir en revistas culturales convencionales.

El Ágora de las Mujeres nació de repente. Fue el producto de una reacción rápida y espontánea, un acto de dignidad de un grupo de feministas de Quito ante una entrevista publicada por el vespertino Ultimas Noticias (Quito), el 20 de julio de 2002. En ésta, el Presidente de la Casa de la Cultura Ecuatoriana, Raúl Pérez Torres (escritor), declara al periodista joven que lo entrevista "haberse comido a todas las mujeres de Quito". Unas pocas feministas, cuando nos enteramos, enviamos correos electrónicos a las listas de mujeres del movimiento, a los medios de comunicación y a las filiales de la Casa de la Cultura en todo el Ecuador protestando por el hecho y pidiendo la destitución de su presidente. No imaginamos que íbamos a tener la acogida que tuvimos: fuimos noticia durante unos pocos días, nos abrieron espacios en los programas de mayor audiencia de radios de Quito, aparecieron notas de prensa en diarios, entre ellos en El Universo (Guayaquil), el de mayor circulación nacional, nos ofrecieron espacios en semanarios. Nos desbordaron.

Sobre la marcha tuvimos que nombrarla y diseñarla. El primer mes, durante la campaña "Perecerás" (un juego de palabras con el apellido de Pérez) escribimos diez Ágoras. Luego los envíos fueron espaciándose mientras paulatinamente intentábamos dar forma a este medio que nos había sorprendido a todas. También tuvimos que contener las demandas represadas de muchas mujeres sobre asuntos de violencia y discriminación de género que no paraban de llegar, seguramente porque sintieron que este medio las acogería. En esta identificación de objetivos de repente saltamos de un tema muy específico a la heterogeneidad temática que, según Maria Juracy Filgueiras Toneli², es una de las dificultades de las publicaciones de mujeres. Hemos tratado de resolverla definiendo simultáneamente temas, audiencia y objetivos del medio, a ratos más por descarte que por afirmación. Sabemos lo que no queremos. No queremos convertirla en un espacio de denuncia de un sin fin de actos cotidianos de violencia contra las mujeres; tampoco una plataforma donde las lideresas ejerciten la política formal y partidaria. Queremos tener un espacio propio desde donde debatir sobre las instituciones y las políticas culturales, así como sobre el quehacer de escritores-as, artistas, periodistas, comunicadores, cientistas sociales, científicos-as, mundos todos ellos prácticamente impermeables a los discursos de las mujeres, las feministas y el género en el Ecuador. Queremos usar la palabra para provocar, incitar a la desnaturalización de los imaginarios androcéntricos, de esos puntos de vista centrados en lo masculino que se activan ante cualquier estímulo poniendo en marcha actitudes, comportamientos, estereotipos conservadores sobre el ejercicio de la sexualidad y el poder masculino. Queremos tornar audibles los discursos feministas en el medio cultural e intelectual, erosionar el pensamiento androcéntrico que lo domina. Pero también queremos sostener las referencias feministas dentro de las organizaciones del movimiento de mujeres, entre sus lideresas, bañar de estética a las políticas feministas.

Los temas fueron marcando el camino: la exclusión de las mujeres de las políticas culturales; el cuerpo de las mujeres en el imaginario de escritores y artistas; leer y escribir literatura como mujeres; la violencia de género en las cúpulas políticas; la guerra contra Irak; género y uso del agua; los derechos sexuales de las mujeres; la lluvia de cenizas del volcán Reventador sobre Quito. Todos estos temas surgieron de la coyuntura. Lo que hemos hecho es elegir entre los múltiples acontecimientos que irrumpen en el escenario público convertidos en hechos políticos, aquellos que mejor se prestan para poner en evidencia la diferencia sexual y a las mujeres como productoras de sentidos y conocimientos. Queremos tocar lo simbólico, llegar a los imaginarios, abonar el desaparecimiento de esa división ancestral entre 'las que dan vida' y 'los que dan sentido' de la que habla Julia Kristeva en su carta a Catherine Clément $^{3}$. 


\section{La audiencia}

Caracola está dirigida a académicas y estudiantes universitarias-os. Sin embargo no fue esta la audiencia que tenía en mente Raquel Rodas-Morales cuando fundó la revista. Más bien pensó en las lideresas y profesionales del movimiento de mujeres. Con tristeza dice haber constatado que son las que menos leen y buscan nuevas ideas. Siguen aferradas a los conceptos del marxismo, el sindicalismo y, más recientemente, de los derechos. Reclaman la reflexión, pero en la práctica no se conectan con las propuestas estéticas, literarias y artísticas, y sólo eventualmente se apoyan en las académicas feministas, aunque no las leen. Este es el primer y gran problema que enfrenta Caracola, añade Raquel Rodas-Morales: "hemos imaginado un público lector, pero ese público todavía no nos ha imaginado."

No conocemos, apenas sospechamos por la lista de distribución, al público lector de El Ágora. Durante la campaña "Perecerás" recogimos algunas pistas sobre los motivos de la gran acogida que tuvo: habíamos ocupado un nicho vacío. Nuestras críticas calaron en hombres y mujeres sobre todo jóvenes, porque las expresiones del presidente de la Casa de la Cultura les avergonzaron. El Ágora también canalizó el silencioso descontento de muchos intelectuales con respecto al funcionamiento y orientación de la principal institución cultural del país: La Casa de la Cultura Ecuatoriana.

\section{Medios fronterizos de acción políitica feminista}

Tanto Caracola como El Ágora de las Mujeres están dirigidas por dos feministas articuladas al movimiento de mujeres y a la academia, radicadas en Quito. Ambas practicamos el ensayo y la critica literaria. Las colaboradoras de los dos medios son escritoras, poetas y artistas no necesariamente feministas, así como feministas que se mueven en los dos espacios: la academia y el movimiento. Poniendo el acento en los cuerpos sexuados, el objetivo de Caracola y El Ágora de las Mujeres es introducir puntos de vista feministas entre intelectuales y artistas, a la vez que mejorar los vínculos entre las académicas y las activistas, entre la teoría y la política feministas; aligerar el pensamiento académico y valerse de las investigaciones hechas en este espacio; enriquecer la diversidad ensayando y recreando múltiples cruces: género- étnia, género-generación, género- sexualidad, género-religión, género región y otros tantos posibles.

No nacieron dentro de una ONG ni de una Universidad, no son iniciativas institucionales como ocurre con muchas publicaciones lideradas por mujeres articuladas al movimiento ${ }^{4}$. Son iniciativas personales que se sitúan en las fronteras de lo cultural, lo académico, lo cotidiano, lo político, lo coyuntural. No son únicamente medios de formación y de información. Son ante todo, como sugiere Jacira Melo ${ }^{5}$, propuestas de renovación de los discursos políticos sobre la condición de las mujeres, espacios para divulgar ideas, preguntas, conceptos, teorías feministas que provoquen la polémica, la discusión, el debate: su objetivo principal es, ante todo, situar la acción política feminista. Efectivamente, El Ágora de las Mujeres y Caracola son espacios de legitimación de las voces feministas.

\section{La búsqueda de audibilidad}

Desde fines de la década de 1990, cuando la crisis económica y política se agudizó en el Ecuador, cuando se adoptó el dólar de EU como moneda oficial, las voces de las lideresas del movimiento de mujeres, los discursos de las activistas y feministas o no se escuchan, o se escuchan de vez en cuando en los escenarios públicos donde compiten con otros discursos, muy sonoros estos sí, como los de los indígenas y el de las elites de la 
ciudad de Guayaquil. La audibilidad de los discursos de esas lideresas es también muy débil entre mujeres y hombres científicos, escritores y artistas, entre las elites empresariales, entre comunicadores y entre las autoridades de la iglesia católica, es decir, entre los grupos sociales poderosos del Ecuador ${ }^{6}$. A la indudable visibilidad lograda en la década de 1990 por las mujeres, a través de su presencia ocupando importantes espacios de poder en el Estado y en la sociedad civil, no ha acompañado la audibilidad ${ }^{7}$, palabras nuevas, formas nuevas, símbolos que pongan en jaque a los imaginarios androcéntricos, es decir, aquellos que adoptan el punto de vista masculino como central y relegan a los márgenes a todo cuanto se considera impertinente para valorar como superior esa perspectiva.

Sospechamos que en todo eso tiene mucho que ver el paulatino silenciamiento de los feminismos, su desvanecimiento en el Ecuador. Los discursos de las lideresas ponen el acento en las injusticias económicas y en las luchas por la redistribución de la riqueza material. Pocas conocen la genealogía feminista, ninguna coloca en su agenda temas relacionados con la producción y consumo de ciencia, literatura y arte $^{8}$. Nos entristece constatar que actualmente la discusión feminista parecería sobrar, parecería que se ha perdido de vista, que estos discursos son luchas por el sentido, son prácticas que persiguen representar la vida de otra manera.

Mientras institucionalizaban el enfoque de género, durante la década de 1990 las lideresas dejaron de lado ese compromiso fundacional de los feminismos. Mientras pugnaban por ocupar los escenarios políticos y extra-domésticos, silenciaban problemas que afectan las prácticas interpersonales y que están directamente relacionados con la simbolización de la diferencia sexual.

\section{Las lecciones y los desafíos}

El Ágora de las Mujeres y Caracola se sostienen por la tenacidad y el trabajo no remunerado de unas pocas mujeres persistentes, lo cual tiene un límite. Hasta fines del 2003 habían circulado once revistas y 22 páginas virtuales.

Caracola ha recibido financiamientos parciales para algunos números, insuficientes para pagar a las colaboradoras, mientras que la imprenta sigue subsidiada. Para la producción, la directora cuenta con el apoyo de estudiantes o egresadas de comunicación de tres universidades de Quito. Se acercaron espontáneamente, como lectoras, y solicitaron hacer sus prácticas profesionales; algunas terminaron quedándose e interesándose por el feminismo. Recogen información, elaboran artículos bajo la supervisión de Raquel.

Pese a que los costos y esfuerzos de producción de El Ágora son menores que los de Caracola, que es gratuita y fácil de distribuir, su circulación ha sido muy irregular pues depende del tiempo que las editoras puedan destinar a escribir y a encontrar colaboradoras. Como no hemos conseguido ni siquiera financiamientos parciales, actualmente está en un obligado receso.

La supervivencia de ambos medios depende de la llegada (o no) de financiamiento. La búsqueda ha sido constante y los resultados pobres, probablemente porque la orientación de ambas publicaciones no es atractiva ni para la empresa privada, ni para el Estado, ni para los medios masivos de comunicación social, ni para las agencias de cooperación internacional que apoyan a las organizaciones de mujeres en el Ecuador. Y como la opción ha sido desde el inicio "no dorar la píldora", las dificultades son mayores que las de otros medios de comunicación, tal como lo relata Fe Pompeu?:

O feminismo trabalha com temas polêmicos, 'fortes'. Por exemplo, apregoa o direito ao aborto ou o direito a amar pessoas do mesmo sexo. Convenhamos que esses assuntos 
não são iguais a propagandear dietas alimentares, tratamentos para eliminar a barriga ou dicas de como 'fisgar' o partidão.

Nas publicações comerciais, as polêmicas se dividem entre as que podem aparecer e as que não devem aparecer. Por exemplo, denúncias de corrupção e nepotismo são bem aceitas. Mas quando as mulheres decidem sair do seu lugar e reivindicar direitos e equidade, a polêmica fica polêmica demais. Aborto, liberdade sexual, remuneração igual para tarefa igual, representatividade política etc. águam a caipirinha brasileira das tradições. As editoras e redatoras das publicações feministas seguram a batata quente dos temas difíceis e, ao mesmo tempo, descascam o abacaxi para popularizar as idéias do feminismo.

Pero inclusive con financiamiento persisten problemas de otra índole. Uno es que la mayoría de las lideresas maduras y de las activistas de las organizaciones del movimiento de mujeres no lee. El otro: hay pocas feministas que hayan hecho de la escritura un oficio, y pocas mujeres periodistas, escritoras, ensayistas que estén interesadas en el feminismo. La mayoría busca el reconocimiento de los escritores, intelectuales y artistas con prestigio que transitan por los círculos de poder. Faltan mujeres dispuestas a traicionar a sus maestros. Ojalá que esta descomunal tarea no nos obligue a descansar sobre los hombros de profesionales especializadas-os, tal como parece ser el camino elegido por algunas publicaciones feministas en Brasil $^{10}$, antes que invertir en la profesionalización de las editoras y colaboradoras.

Si a través de Caracola y El Ágora de las Mujeres pretendemos elevar la audibilidad de los discursos feministas en los medios masivos de comunicación social y en los espacios académicos, literarios y artísticos, necesitamos provocar un doble movimiento: que mujeres, comunicadoras, escritoras, artistas y científicas se apropien de esta página virtual y también que las lideresas del movimiento la lean y se animen a escribir.

¿Cómo lograr que las comunicadoras, escritoras, ensayistas y artistas sean tocadas por lo político, y que las lideresas sean rozadas por lo estético?

¿Cómo promover alianzas con comunicadores, mujeres y hombres, que trabajan en los grandes medios masivos de comunicación (prensa, radio, TV) y que están cargo de programas y páginas editoriales con gran audiencia y legitimidad, con el fin de elevar la audibilidad de los discursos feministas?

¿Cómo provocar a intelectuales, escritores y artistas, con el fin de erosionar el pensamiento androcéntrico que domina las ciencias, la literatura y el arte en el Ecuador?

Quito, marzo 1, 2004

\section{Notas}

Copyright (C) 2004 by Revista Estudos Feministas.

' La información sobre Caracola se basa en la entrevista que realicé a Raquel Rodas en noviembre de 2003 en Quito.

2 Maria Juracy F. TONELI, 2003, 268.

${ }^{3}$ Catherine CLÉMENT y Julia KRISTEVA, 2000, p. 23.

${ }^{4}$ Ver Dossier "Publicações Feministas Brasileiras: Compartilhando Experiências" en Revista Estudos Feministas, vol. 11, no. 1, p. 217-306, 2003

5 Jacira MELO, 2003, p. 298.

- Silvia VEGA, Cuvi y Alexandra MARTínEZ, 2001 ; Cuvi 2003; Raquel RODAS-MORALES, 2003.

${ }^{7}$ Este argumento sobre la audibilidad lo desarrollo y profundizo sobre todo en la ponencia "Melismas o el tono de los discursos de género en el Ecuador", ponencia que presenté en el Seminario Internacional "Balance de los estudios de género en el área andina", organizado por el British Council, CLACSO y la Red para el Desarrollo de las Ciencias Sociales de la Pontificia Universidad Católica del Perú. El Seminario se realizó en Lima, en septiembre de 2003.

${ }^{8}$ RODAS-MORALES, 2002 
${ }^{9}$ Fe POMPEU, 2003, 305.

${ }^{10}$ TONELI, 2003, p. 266.

\section{Fuentes}

CLÉMENT, Catherine y KRISTEVA, Julia. Lo femenino y lo sagrado. Madrid: Ediciones Cátedra, 2000.

CUVI-SÁNCHEZ, María. "Melismas o los tonos de los discursos de género en el Ecuador." Ponencia presentada en el Seminario Internacional "Balance de los estudios de género en el área andina", organizado por el British Council, CLACSO y la Red para el Desarrollo de las Ciencias Sociales de la Pontificia Universidad Católica del Perú, Lima, septiembre de 2003.

. "Disonancias entre las elites empresariales a principios del siglo XXI". In: BRETÓN, Víctor y GARCÍA, Francisco (eds.) Estado, etnicidad y movimientos sociales en América Latina. Ecuador en crisis. Barcelona: Icaria, 2003. p. 277-317.

TONELI, Maria Juracy Filgueiras. "Publicações Feministas Sediadas em Ongs: limites alcances e possibilidades". Revista Estudos Feministas, vol. 11, n 1, 2003, p. 265-270.

MELO, Jacira. "Publicar é uma ação política". Revista Estudos Feministas, vol. 11, n. 1 , 2003, p. 298-301.

POMPEU, Fe. "O público, esse desconhecido". Revista Estudos Feministas, vol. 11 , n. 1, p. 305-307, 2003.

RODAS-MORALES, Raquel. "Textos, tonos y texturas. Discursos de comunicadoras y comunicadores sobre el género y la crisis en el Ecuador". Quito: Fondo para la lgualdad de Género de ACDI, 2002. Documento de trabajo N5. Proyecto 003 "Cómo nos miran propias y ajenos".

"Muchas voces, demasiados silencios. Los discursos de las lideresas del movimiento de mujeres del Ecuador". Quito, Fondo para la Igualdad de Género de ACDI, 2002. Documento de Trabajo №4. Proyecto 003 "Cómo nos miran propias y ajenos".

VEGA, Silvia, CUVI-SÁNCHEZ, Maria y MARTíNEZ, Alexandra. Género y ciencia. Los claroscuros de la investigación científica y tecnológica en el Ecuador. Quito: FUNDACYT y AbyaYala, 2001.

\section{Feminist's Publications in El Ecuador: Caracola $y$ El Ágora de las Mujeres}

Abstract: This essay is about Caracola y El Ágora de las Mujeres, two feminist's publications that circulate in Ecuador. The first is a published paper journal that comes out every three months, it first appeared in March 2001; the second was as on-line webzine that appeared between 2002 and 2003. This essay talks about the objectives of both these publications, it describes their reception and the challenges they fac.e The author's intentions are twofold: how to make feminist discourses heard in Ecuador and how to erode the androcentric line of thought that dominates the sciences, literature and art in this country.

Key Words: feminist's publications, feminist's politics, women's movements 